Sri Lankan Journal of Physics, Vol. 21 (2020) 11-36

http://doi.org/10.4038/sljp.v21i1.8069

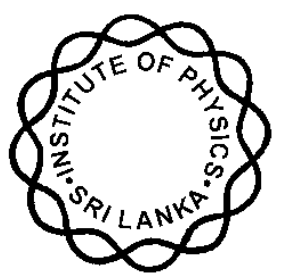

INSTITUTE OF PHYSICS - SRI LANKA

Research Article

\title{
A theoretical investigation of nonrelativistic bound state solution at finite temperature using the sum of modified Cornell plus inverse quadratic potential
}

\section{Abdelmadjid Maireche*}

Laboratory of Physics and Material Chemistry, Physics Department, Sciences Faculty, University of M'sila- Algeria

\begin{abstract}
New modified nonrelativistic bound state energy eigenvalues have been obtained for the hydrogenic atoms with spin $1 / 2$ under the sum of modified Cornell plus inverse quadratic potential (MCIQP), at finite temperature, in the symmetries of the noncommutative threedimensional real space phase (NC: 3D-RSP). The ordinary sum of Cornell plus inverse quadratic potential is extended by including new central terms to become MCIQP. In addition, MCIQP is suggested as a quark-antiquark interaction potential for studying the masses of heavy and heavy-light mesons in (NC: 3D-RSP), in which the potential satisfies the features of quantum chromodynamics theory of strong interaction. For this purpose, the modified radial Schrödinger equation is analytically solved using the generalized Bopp's shift method and standard perturbation theory. The energy eigenvalues and the corresponding new Hamiltonian operator are obtained in (NC: 3D-RSP). These results are applied to calculate the mass of mesons such as charmonium $c \bar{c}$, bottomonium $b \bar{b}$ and mesons $\bar{c}$ with spin (0 or 1$)$. In a thermal medium of a positive temperature, the new parameters of the studied potential MCIQP become temperature dependent because of color
\end{abstract}

\footnotetext{
* Corresponding author: abmaireche@gmail.com
}

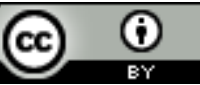

https://orcid.org/0000-0002-8743-9926 
screening. It is found that the perturbative solutions of the discrete spectrum can be expressed on the Gamma function, the discreet atomic quantum numbers $(j, l, s, m)$ and the potential parameters $(C, G, F, L)$, in addition to noncommutativity parameters ( $\Theta$ and $\bar{\theta})$. The influence of the induced magnetic field and the coupling parameter of the spin field on some quantum properties of the system have also been studied. The total complete degeneracy of modified energy levels under MCIQP is found to be equal to $4 n^{2}$, which gives a very good indicator that our new treatments produce clear energy values when compared with similar energy levels obtained in ordinary relativistic quantum mechanics (RQM). New mass spectra for the quarkonium systems is found to be equal to the sum of ordinary values in RQM plus two perturbative terms proportional to the parameters $(\Theta$ or $\chi)$ and $(\bar{\theta}$ or $\bar{\sigma})$ of noncommutativity space-phase. These results are in good agreement with the already existing results in nonrelativistic noncommutative quantum mechanics (NRNCQM) where the physical treatment was done using other potentials such as new modified potential containing Cornell, Gaussian and inverse square terms and modified quark-antiquark interaction potential.

Keywords: Schrödinger equation, the hydrogenic atoms, the heavy quarkonium systems, Cornell potential, inverse quadratic potential, noncommutative space-phase, the Weyl Moyal star product, the Bopp's shift method.

\section{INTRODUCTION}

Over several decades, there has been a growing interest among researchers to investigate the analytical solutions of the Schrödinger equation for physical potential models in quantum mechanical systems. Nonrelativistic wave equations and energy eigenvalues have been of interest for theoretical physicists in many branches of physics such as nuclear physics, atomic physics and quantum chromodynamics (QCD). Recently, in a particular case, the study of different properties of heavy-light mesons was very important for understanding the structure of hadrons and the dynamics of heavy quarks in QCD theory. Very recently, considerable efforts have been made to understand different properties of heavy-light mesons; so researchers calculated the mass spectra of quarkonium systems such as charmonium, bottomonium and mesons $c \bar{s}$ with the quark-antiquark interacted under Cornell potential, mixing between the Cornell and the harmonic oscillator potential, the quadratic and Morse potential in the context of relativistic and non-relativistic quantum mechanics ${ }^{1-5}$. This work is motivated by several recent studies such as the nonrenormalisation of the standard model, string theory, quantum gravity; nonrelativistic noncommutative quantum mechanics (NRNCQM) that has attracted much attention ${ }^{6-10}$. The noncommutativity of space-phase was initially used by W. Heisenberg ${ }^{11}$ in 1930 and was formalized by H. Snyder ${ }^{12}$ in 1947 . It should be noted that nonrelativistic quantum mechanics is included within the framework of NRCQM. The main objectives of this work are to further develop the work done by A. I. Ahmadov et al. ${ }^{1}$, expand it to the symmetries of NRNCQM, and to achieve a more accurate physical description so that this study becomes 
valid in the field of nanotechnology. Furthermore, attempts are made to find new applications including solutions of the modified radial Schrödinger equation with a class of Cornell potentials influenced by the temperature. On the other hand, more profound interpretations are sought in the sub-atomics and nano-scales using an updated version of the Cornell plus inverse quadratic potential at finite temperature, which has the following new form:

$$
V_{c i q}(r)=A(T, r) r-\frac{B(T, r)}{r}+C(T, r) r^{2} \rightarrow V_{c i q}(\hat{r})=V_{c i q}(r)-\left(\frac{C}{r^{4}}+\frac{G}{2 r^{3}}+\frac{F}{2 r}-L\right) \overrightarrow{\mathbf{L}} \vec{\Theta}
$$

Here $A(T, r), B(T, r)$ and $C(T, r)$ are three parameters dependent on the temperature and the interquark distance $r$ (see Eq. (8)). The parameters $C, G, F$ and $L$ are determined in Eq. (10) while the coupling $\overrightarrow{\mathbf{L}} \vec{\Theta}$ is interpreted as the interaction between the angular momentum operator and noncommutativity properties of space-phase (see Eq. (16)). In this work, nonrelativistic quantum dynamics of the hydrogenic atoms such as $\left(\mathrm{He}^{+}, \mathrm{Be}^{+}\right.$and $\left.\mathrm{Li}^{2+}\right)$ are studied with spin-1/2 by solving the MSE using the generalized Bopp's shift method and standard perturbation theory in (NC: 3D-RSP). Furthermore, this study can be generalized to describe the quarkonium systems such as charmonium $c \bar{c}$, bottomonium $b \bar{b}$ and mesons $c \bar{s}$ at finite temperature with spin-(0 or 1) in NRNCQM symmetries. The new structure of NRNCQM based on new canonical commutative relations in both the Schrödinger and the Heisenberg pictures (SP and HP), are as follows ${ }^{13-21}$ (Throughout this paper, the natural units $c=\hbar=1$ will be used):

$$
\begin{aligned}
& {\left[\hat{x}_{\mu}{ }^{*}, \hat{p}_{v}\right]=\left[\hat{x}_{\mu}(t), \hat{p}_{v}(t)\right]=i \delta_{\mu v},\left[\hat{x}_{\mu}{ }^{*}, \hat{x}_{v}\right]=\left[\hat{x}_{\mu}(t)^{*}, \hat{x}_{v}(t)\right]=i \theta_{\mu v}} \\
& \text { and }\left[\hat{p}_{\mu}{ }^{*}, \hat{p}_{v}\right]=\left[\hat{p}_{\mu}(t), \hat{p}_{v}(t)\right]=i \bar{\theta}_{\mu v}
\end{aligned}
$$

where the indices $(\mu, v \equiv 1,2,3)$ while $\left[A^{*}, B\right] \equiv A^{*} B-B^{*} A$, for any two operators $A$ and $B$. However, the new operators $\hat{\xi}(t)=\hat{x}_{\mu}(t)$ or $\hat{p}_{\mu}(t)$ in HP depend on the corresponding timeindependent operator $\hat{\xi}=\hat{x}_{\mu}$ or $\hat{p}_{v}$ in Spas shown in the following generalized projections relations:

$$
\begin{aligned}
& \xi(t)=\exp \left(i \hat{H}_{c i q}\left(t-t_{0}\right)\right) \xi \exp \left(-i \hat{H}_{c i q}\left(t-t_{0}\right)\right) \Rightarrow \\
& \xi(t)=\exp \left(i \hat{H}_{n c}^{c i q}\left(t-t_{0}\right)\right) * \hat{\xi} * \exp \left(-i \hat{H}_{n c}^{c i q}\left(t-t_{0}\right)\right)
\end{aligned}
$$

here $\xi=x_{\mu}$ or $p_{\mu}$ and $\xi(t)=x_{\mu}(t)$ or $p_{\mu}(t)$. The evaluations of the dynamics systems are described from the following generalized motion equations in NRNCQM: 


$$
\frac{\mathrm{d} \xi(t)}{\mathrm{dt}}=\left[\xi(t), \hat{H}_{c i q}\right]+\frac{\partial \xi(t)}{\partial \mathrm{t}} \Rightarrow \frac{\mathrm{d} \hat{\xi}(t)}{\mathrm{dt}}=\left[\hat{\xi}(t), \hat{H}_{n c}^{c i q}\right]+\frac{\partial \hat{\xi}(t)}{\partial \mathrm{t}}
$$

where $\hat{H}_{c i q}$ and $\hat{H}_{n c}^{c i q}$ represent the quantum Hamiltonian operators for CIQP and MCIQP in the NRQM and its extension, respectively. The very small two parameters $\theta^{\mu v}$ and $\bar{\theta}^{\mu v}$ (compared to the energy) are elements of two antisymmetric real matrixes with dimensions of (length) ${ }^{2}$ and (momentum) ${ }^{2}$, respectively. In addition, $(*)$ denotes to the Weyl Moyal star product, which is generalized between two arbitrary functions $(f, g)(x, p)$ to the new form $\hat{f}(\hat{x}, \hat{p}) \hat{g}(\hat{x}, \hat{p}) \equiv(f * g)(x, p)$ in (NC: 3D-RSP) symmetries ${ }^{22-31}$ as follows:

$$
(f g)(x, p) \rightarrow(f * g)(x, p) \approx\left(f g-\frac{i}{2} \theta^{\mu v} \partial_{\mu}^{x} f \partial_{v}^{x} g-\frac{i}{2} \bar{\theta}^{\mu v} \partial_{\mu}^{p} f \partial_{\nu}^{p} g\right)(x, p)
$$

The second and the third terms in the above equation present the effects of (space-space) and (phase-phase) noncommutativity properties. The purpose of this work is to solve the modified radial Schrödinger equation for the MCIQP model at finite temperature in (NC: 3D-RSP) symmetries using the generalized Bopp's shift method and standard perturbation theory. The organization scheme of the present work is given as follows: In the next section, we briefly review the ordinary SE with CIQP. Section 3 is devoted to study MSE by applying the generalized Bopp's shift method and the standard perturbation theory. This is to find the quantum spectrum of the perturbed spin-orbital operator and induced Zeeman effect for hydrogenic atoms such as $\mathrm{He}^{+}, \mathrm{Be}^{+}$and $\mathrm{Li}^{2+}$ under MCIQP at finite temperature. In the next section, we determine the energy spectra of the quarkonium systems under MCIQP, in addition to the new formula of mass spectra in (NC: 3D-RSP) symmetries. The main results of this work will be presented in the sixth section. Finally, in section 7, this paper is concluded with some brief remarks.

\section{OVERVIEW OF THE EIGENFUNCTIONS AND THE ENERGY EIGENVALUES FOR SE UNDER CIQP}

The Schrödinger equation (SE) for the sum of Cornell plus inverse quadratic potential (CIQP) at a finite temperature is of the form ${ }^{1}$ :

$$
V(r)=A r-\frac{B}{r}+C r^{2} \rightarrow V_{c i q}(r)=A(T, r) r-\frac{B(T, r)}{r}+C(T, r) r^{2}
$$

The interaction potential between a quark and anti-quark is determined from the standard Cornell potential $V(r)=A r-\frac{B}{r}$. The first part is responsible for quark confinement at large distances while the second dominates at short distances ${ }^{4}$. This potential has been extensively studied in both relativistic and non-relativistic quantum mechanics and has attracted a great 
deal of attention in particle physics. Here $A$ and $B$ are two positive coefficients and $\mathrm{r}$ is the interquark distance. As it is mentioned in the first reference, the color screening in a thermal medium of a positive temperature $T$ can modify the potential to become dependent on temperature, which can be parameterized in the following form ${ }^{1}$ :

$$
V_{T}(r)=\frac{A}{\mu_{D}(T) r}\left(1-\exp \left(-\mu_{D}(T)\right)\right)-\frac{B}{r} \exp \left(-\mu_{D}(T) r\right)+\frac{C}{r^{2}} \exp \left(-\mu_{D}(T) r\right)
$$

with

$$
A(T, r)=\frac{A}{\mu_{D}(T) r}\left(1-\exp \left(-\mu_{D}(T) r\right)\right), \quad B(T, r)=B \exp \left(-\mu_{D}(T) r\right) \text { and } C(T, r)=C \exp \left(-\mu_{D}(T) r\right)
$$

Here $\mu_{D}(T)$ is the Debye screening mass. Expanding with the Taylor series expansion around $r=0$, the potential in Eq. (7) can be simplified to the new form ${ }^{1}$ :

$$
V(r)=D+F r-\frac{G}{r}-L r^{2}-\frac{C}{r^{2}}
$$

with

$$
\begin{aligned}
& D=B \mu_{D}(T)+1 / 2 C \mu_{D}{ }^{2}(T), \mathrm{F}=\mathrm{A}-1 / 2 B \mu_{D}{ }^{2}(T), \quad G=B+\mu_{D}(T), \mathrm{L}=1 / 2 A \mu_{D}(T) \\
& B(T, r)=B \exp \left(-\mu_{D}(T) r\right) \text { and } C(T, r)=C \exp \left(-\mu_{D}(T) r\right)
\end{aligned}
$$

It is well known for the physicists, that studying any physical quantum system requires solving the original SE given as ${ }^{32-33}$

$$
\left(\frac{P^{2}}{2 \mu}+V(r)\right) \Psi_{n l m}(r, \theta, \varphi)=E_{n l} \Psi_{n l m}(r, \theta, \varphi)
$$

where $E_{n l}$ is the energy, $\mu=\frac{m_{e} m_{Z e}}{m_{e}+m_{Z e}}$ is the reduced mass of the hydrogenic atom $\left(m_{e}\right.$ and $m_{Z e}$ represent the masses of the electron e and the atom Ze, respectively). In addition, for the quarkonium systems, the reduced mass is $\frac{m_{q} m_{\bar{q}}}{m_{q}+m_{\bar{q}}}$. The separation of coordinates in the complete wave function is $\Psi(r, \theta, \varphi)=\frac{\chi(r)}{r} Y_{l}^{m}(\theta, \varphi)$, where $Y_{l}^{m}(\theta, \varphi)$ is the spherical harmonic function. If the radial part $U_{n l}(r)=\frac{\chi(r)}{r}$ is inserted into the SE, one can get the radial part in two forms as follows:

$$
\frac{d^{2} U_{n l}(r)}{d r^{2}}+\frac{2}{r} \frac{d U_{n l}(r)}{d r}+2 \mu\left(E_{n l}-V_{e f f}^{c i q}\right) U_{n l}(r)=0 \Rightarrow \frac{d^{2} \chi_{n l}(r)}{d r^{2}}+2 \mu\left(E-V_{e f f}^{c i q}\right) \chi_{n l}(r)=0
$$

Here $V_{e f f}^{\text {ciq }}=D+F r-\frac{G}{r}+L r^{2}+\frac{C}{r^{2}}+\frac{l(l+1)}{2 \mu r^{2}}$. The complete wave function and the corresponding eigenvalues of the SE for the potential in Eq. (9) are given by ${ }^{1}$ :

$$
\Psi(r, \theta, \varphi)=C_{n l} r^{-\frac{N}{2 \sqrt{H_{n}}}} \exp \left(\sqrt{H_{n}} r\right)\left(-r^{2} \frac{d}{d r}\right)^{n}\left(r^{-2 n-\frac{N}{\sqrt{H_{n}}}} \exp \left(-2 \sqrt{H_{n}} r\right)\right) Y_{l}^{m}(\theta, \varphi)
$$

and 


$$
E_{n l}=D+\frac{3 F}{\delta}-\frac{6 L}{\delta^{2}}-\frac{1}{2 \mu}\left[\frac{2 \mu\left(\frac{3 F}{\delta^{2}}-\frac{8 L}{\delta^{3}}+G\right)}{(1+2 n) \pm \sqrt{1+4 l(l+1)+\frac{8 \mu F}{\delta^{3}}-24 \frac{L}{\delta^{4}}+8 \mu C}}\right]^{2}
$$

where $H_{n}=-2 \mu\left(E_{n}-D-3 F r_{0}+6 L r_{0}^{2}\right), N=2 \mu\left(3 F r_{0}{ }^{2}-8 L r_{0}^{3}+G\right)$ and $r_{0} \equiv 1 / \delta$ characterize the radius of meson while $C_{n l}$ is the normalization constant.

\section{SOLUTION OF MSE FOR MCICP}

\subsection{Review of the generalized Bopp's shift method}

In this subsection, an overview or a brief preliminary for MCIQP in (NC: 3D-RSP) symmetries is presented. To perform this task with the physical form of MSE, it is necessary to replace the ordinary Hamiltonian operator $\hat{H}\left(x_{\mu}, p_{\mu}\right)$, ordinary complex wave function $\Psi(\vec{r})$ and ordinary energy $E_{n l}$ by the new Hamiltonian operator $\hat{H}_{n c}^{c i q}\left(\hat{x}_{\mu}, \hat{p}_{\mu}\right)$, new complex wave function $\Psi(\overrightarrow{\hat{r}})$ and new values of energy $E_{n c}^{c i q}$, respectively. Replacing the ordinary product by the Weyl Moyal star product, the MSE in (NC-3D: RSP) symmetries can be constructed as follows ${ }^{34-40}$ :

$$
\hat{H}_{c i q}\left(x_{\mu}, p_{\mu}\right) \Psi(\vec{r})=E_{n l} \Psi(\vec{r}) \Rightarrow \hat{H}_{n c}^{c i q}\left(\hat{x}_{\mu}, \hat{p}_{\mu}\right) * \Psi(\overrightarrow{\hat{r}})=E_{n c}^{c i q} \Psi(\overrightarrow{\hat{r}})
$$

The Bopp's shift method ${ }^{41-45}$ has been successfully applied to RNCQM and NRNCQM problems using modified Dirac equation (MDE), modified Klein-Gordon equation (MKGE) and MSE. This method has produced very promising results for number of situations having a physical and chemical interests. The method reduces MDE, MKGE and MSE to the Dirac equation, the Klein-Gordon equation and SE, respectively under two simultaneously translations in space and phase $\left(x_{\mu} \rightarrow \hat{x}_{\mu} \equiv x_{\mu}-\frac{\theta_{\mu \nu}}{2} p_{\nu}\right.$ and $\left.\hat{p}_{\mu}=p_{\mu}+\frac{\bar{\theta}_{\mu \nu}}{2} x_{v}\right)$ in RQM and NRQM. It is based on the following new commutators $22-28$ :

$$
\left[\hat{x}_{\mu}, \hat{x}_{v}\right]=\left[\hat{x}_{\mu}(t), \hat{x}_{v}(t)\right]=i \theta_{\mu \nu} \text { and }\left[\hat{p}_{\mu}, \hat{p}_{v}\right]=\left[\hat{p}_{\mu}(t), \hat{p}_{v}(t)\right]=i \bar{\theta}_{\mu \nu}
$$

The new coordinates $\left(\hat{x}_{\mu}, \hat{p}_{v}\right)$ in (NC: 3D-RSP) symmetries are defined in terms of the corresponding commutative counterparts $\left(x_{\mu}, p_{v}\right)$ in NRQM via, as follows $20-26$ :

$$
\left(x_{\mu}, p_{v}\right) \Rightarrow\left(\hat{x}_{\mu}, \hat{p}_{v}\right)=\left(x_{\mu}-\frac{\theta_{\mu v}}{2} p_{v}, p_{\mu}+\frac{\bar{\theta}_{\mu v}}{2} x_{v}\right)
$$


The above equation allows us to obtain the operators $\left(\hat{r}^{2}, \hat{p}^{2}\right)$ in (NC-3D: RSP) symmetries $^{36-41}$ as follows:

$$
\left(r^{2}, p^{2}\right) \Rightarrow\left(\hat{r}^{2}=r^{2}-\overrightarrow{\mathbf{L}} \vec{\Theta}, \hat{p}^{2}=p^{2}+\overrightarrow{\mathbf{L}} \stackrel{\vec{\theta}}{)}\right)
$$

Where the two couplings $\overrightarrow{\mathbf{L}} \vec{\Theta}$ and $\overrightarrow{\mathbf{L}} \overrightarrow{\bar{\theta}}$ in Eq. (16.1) are given as follows:

$$
\overrightarrow{\mathbf{L}} \vec{\Theta}=L_{x} \Theta_{12}+L_{y} \Theta_{23}+L_{z} \Theta_{13} \quad \text { and } \quad \overrightarrow{\mathbf{L}} \vec{\theta}=L_{x} \bar{\theta}_{12}+L_{y} \bar{\theta}_{23}+L_{z} \bar{\theta}_{13}
$$

here $\left(L_{x}, L_{y}\right.$ and $L_{z}$ ) are just the components of angular momentum operator $\overrightarrow{\mathbf{L}}$ while the element of the antisymmetric matrix $\Theta_{\mu \nu}$ equals $\theta_{\mu \nu} / 2$. Thus, the reduced SE (without star product) can be written as:

$$
\hat{H}_{n c}^{c i q}\left(\hat{x}_{\mu}, \hat{p}_{\mu}\right) * \Psi(\overrightarrow{\hat{r}})=E_{n c}^{c i q} \Psi(\overrightarrow{\hat{r}}) \Rightarrow H_{c i q}\left(\hat{x}_{\mu}, \hat{p}_{\mu}\right) \Psi(\vec{r})=E_{n c}^{c i q} \Psi(\vec{r})
$$

The new Hamiltonian operator $H_{c i q}\left(\hat{x}_{\mu}, \hat{p}_{\mu}\right)$, can be expressed as:

$$
H\left(x_{\mu}, x_{\mu}\right) \Rightarrow H_{n c}^{c i q}\left(\hat{x}_{\mu}, \hat{p}_{\mu}\right) \equiv H\left(\hat{x}_{\mu}=x_{\mu}-\frac{\theta_{\mu v}}{2} p_{v}, \hat{p}_{\mu}=p_{\mu}+\frac{\bar{\theta}_{\mu v}}{2} x_{v}\right)=\frac{\hat{p}^{2}}{2 \mu}+V_{c i q}\left(\hat{r}=\sqrt{\left(x_{\mu}-\frac{\theta_{\mu v}}{2} p_{v}\right)\left(x_{\mu}-\frac{\theta_{\mu v}}{2} p_{v}\right)}\right)
$$

The extended Cornell plus inverse quadratic potential, in (NC: 3D-RSP) symmetries, is suggested as follows:

$$
V_{c i q}(r) \Rightarrow V_{c i q}(\hat{r})=D+F \hat{r}-\frac{G}{\hat{r}}-L \hat{r}^{2}-\frac{C}{\hat{r}^{2}}
$$

Again, Eq. (16.1) is applied to obtain the important three terms $\left(F \hat{r},\left(-\frac{G}{\hat{r}}\right)\right.$ and $\left.\left(-\frac{C}{\hat{r}^{2}}\right)\right)$, which will be used to determine the MCIQP as:

$$
\left\{\begin{array}{l}
-\frac{G}{r} \rightarrow-\frac{G}{\hat{r}}=-\frac{G}{r}-\frac{G}{2 r^{3}} \overrightarrow{\mathbf{L}} \vec{\Theta}+O\left(\Theta^{2}\right), \quad F r \rightarrow F \hat{r}=F r-\frac{F}{2 r} \overrightarrow{\mathbf{L}} \vec{\Theta}+O\left(\Theta^{2}\right) \\
-L r^{2} \rightarrow-L \hat{r}^{2}=-L r^{2}+L \overrightarrow{\mathbf{L}} \vec{\Theta}+O\left(\Theta^{2}\right) \text { and }-\frac{C}{r^{2}} \rightarrow-\frac{C}{\hat{r}^{2}}=-\frac{C}{r^{2}}-\frac{C}{r^{4}} \overrightarrow{\mathbf{L}} \vec{\Theta}+O\left(\Theta^{2}\right)
\end{array}\right.
$$

Substituting, Eq. (20) into Eq. (19), the MCIQP in (NC-3D: RSP) symmetries can be obtained as follows:

$$
V_{c i q}(\hat{r})=V(r)-\left(\frac{C}{r^{4}}+\frac{G}{2 r^{3}}+\frac{F}{2 r}-L\right) \overrightarrow{\mathbf{L}} \vec{\Theta}
$$


Thus, the Cornell plus inverse quadratic potential is extended by including new terms proportional with $\left(1 / r^{4}, 1 / r^{3}\right.$ and $\left.1 / r\right)$ to become MCIQP in (NC-3D: RSP) symmetries. Now, by making the substitution Eq. (21) into Eq. (18), the modified Hamiltonian operator $H_{\mathrm{nc}}^{c i q}(\hat{r})$ in (NC: 3D-RSP) symmetries is found to be as follows:

$$
H_{c i q}\left(x_{\mu}, p_{v}\right) \Rightarrow H_{n c}^{c i q}(\hat{r})=H_{c i q}\left(x_{\mu}, p_{v}\right)+H_{\mathrm{per}}^{c i q}(r)
$$

The operator $H_{c i q}\left(x_{\mu}, p_{v}\right)$ is just the ordinary Hamiltonian operator in ordinary commutative quantum mechanics:

$$
H_{c i q}\left(x_{\mu}, p_{\mu}\right)=\frac{p^{2}}{2 \mu}+D+F r-\frac{G}{r}-L r^{2}-\frac{C}{r^{2}}
$$

Furthermore, the other part in Eq. (22) is proportional to two infinitesimal parameters $(\Theta$ and $\bar{\theta}$ ). It can be expressed in the following form:

$$
H_{\mathrm{per}}^{c \mathrm{iq}}(r)=-\left(\frac{C}{r^{4}}+\frac{G}{2 r^{3}}+\frac{F}{2 r}-L\right) \overrightarrow{\mathbf{L}} \vec{\Theta}+\frac{\overrightarrow{\mathbf{L}} \vec{\theta}}{2 \mu}
$$

Thus, $H_{\mathrm{per}}^{\text {ciq }}(r)$ can be considered as a perturbation term compared with the parent Hamiltonian operator (the principal part) $H_{c i q}\left(x_{\mu}, p_{\mu}\right)$ in (NC: 3D-RSP) symmetries. On the other hand, if $G=Z e$, the attractive term $\left(-\frac{G}{r}\right)$ becomes a Columbian potential. It allows both $H_{n c}^{c i q}\left(\hat{x}_{\mu}, \hat{p}_{\mu}\right)$ and $H_{c i q}\left(x_{\mu}, p_{\mu}\right)$ as good Hamiltonian candidates to describe the Hydrogenic atoms such as $\mathrm{He}^{+}, \mathrm{Be}^{+}$and $\mathrm{Li}^{2+}$ under the influence of external fields in ordinary quantum mechanics and its extension NRNCQM.

\subsection{The exact modified spin-orbit spectrum for heavy quarkonium systems and hydrogenic atoms under MCIQP model}

In this subsection, the same strategy, which was used exclusively in some of our published scientific works ${ }^{38-43}$ is applied. Under such a particular choice, both couplings ( $\overrightarrow{\mathbf{L}} \vec{\Theta}$ and $\overrightarrow{\mathbf{L}} \vec{\theta})$ are reproduced to the new physical forms $(\gamma \Theta \vec{L} \vec{S}$ and $\gamma \vec{\theta} \vec{L} \vec{S})$, respectively. Thus, the new forms of $H_{\mathrm{so}}^{\mathrm{ciq}}(r, \Theta, \bar{\theta})$ for heavy quarkonium system and hydrogenic atoms under MCIQP model are as follows:

$$
H_{\mathrm{per}}^{\mathrm{ciq}}(r) \rightarrow H_{\mathrm{so}}^{\mathrm{ciq}}(r, \Theta, \bar{\theta}) \equiv \gamma\left\{-\left(\frac{C}{r^{4}}+\frac{G}{2 r^{3}}+\frac{F}{2 r}-L\right)+\frac{\bar{\theta}}{2 \mu}\right\} \vec{L} \vec{S}
$$


here $\Theta=\sqrt{\Theta_{12}{ }^{2}+\Theta_{23}{ }^{2}+\Theta_{13}{ }^{2}}, \bar{\theta}=\sqrt{\bar{\theta}_{12}{ }^{2}+\bar{\theta}_{23}{ }^{2}+\bar{\theta}_{13}{ }^{2}}$ and $\gamma \approx \frac{1}{137}$ is a new constant, which plays the role of the fine structure constant in the electromagnetic interaction or quantum electrodynamics (QED) theory. Two vectors $(\vec{\Theta}$ and $\overrightarrow{\bar{\theta}})$ are chosen parallel to the spin $\vec{S}$ of hydrogenic atoms such as $\mathrm{He}^{+}, \mathrm{Be}^{+}$and $\mathrm{Li}^{2+}$. Furthermore, the above perturbative terms $H_{\text {per }}^{\text {ciq }}(r)$ can be rewritten as following new physical form:

$$
H_{s o}^{c \mathrm{iq}}(r, \Theta, \bar{\theta})=-\frac{\gamma}{2}\left\{\left(\frac{C}{r^{4}}+\frac{G}{2 r^{3}}+\frac{F}{2 r}-L\right) \Theta-\frac{\bar{\theta}}{2 \mu}\right\}\left(\vec{J}^{2}-\vec{L}^{2}-\vec{S}^{2}\right)
$$

where $\vec{J}$ and $\vec{S}$ defines the operators of the total angular momentum and spin of hydrogenic atoms (or heavy quarkonium systems). The operator $\vec{L} \vec{S}$ produce the quantum spin-orbit interaction. ( $H_{\text {so }}^{\text {ciq }}(r, \Theta, \bar{\theta}), \mathrm{J}^{2}, \mathrm{~L}^{2}, \mathrm{~S}^{2}$ and $J_{z}$ ) forms a complete set of conserved physical quantities. In addition, for spin-1/2, the eigenvalues of the spin-orbit coupling operator $\vec{L} \vec{S}$ are $k_{ \pm} \equiv \frac{1}{2}\left\{\left(l \pm \frac{1}{2}\right)\left(l \pm \frac{1}{2}+1\right)+l(l+1)-\frac{3}{4}\right\}$. This, corresponds to two polarities, the first one corresponds to $j=l+1 / 2$ (spin-up) while the second polarity corresponds to $j=l-1 / 2$ (spin-down). Then, a diagonal $(3 \times 3)$ matrix $H_{\text {so }}^{\text {ciq }}$ for MCIQP in (NC: 3D-RSP) symmetries can be formed as follows:

$$
H_{s o}^{c i q}=\left(\begin{array}{ccc}
\left(H_{s o}^{c \mathrm{ciq}}\right)_{11} & 0 & 0 \\
0 & \left(H_{s o}^{\mathrm{ciq}}\right)_{22} & 0 \\
0 & 0 & \left(H_{s o}^{c \mathrm{iq}}\right)_{33}
\end{array}\right) \equiv \operatorname{diag}\left(\left(H_{s o}^{c \mathrm{iq}}\right)_{11},\left(H_{s o}^{c \mathrm{iq}}\right)_{22},\left(H_{s o}^{c \mathrm{iq}}\right)_{33}\right)
$$

The non-null elements $\left(H_{s o}^{c \mathrm{iq}}\right)_{11}$ and $\left(H_{s o}^{c \mathrm{iq}}\right)_{22}$ of a diagonal matrix $H_{s o}^{c \mathrm{iq}}$ are given by:

$$
\begin{aligned}
& \left(H_{\text {so }}^{c \mathrm{qq}}\right)_{11}=-\gamma k_{+}(l)\left\{\left(\frac{C}{r^{4}}+\frac{G}{2 r^{3}}+\frac{F}{2 r}-L\right) \Theta-\frac{\bar{\theta}}{2 \mu}\right\} \text { if } j=l+1 / 2 \\
& \left(H_{s o}^{\text {ciq }}\right)_{22}=-\gamma k_{-}(l)\left\{\left(\frac{C}{r^{4}}+\frac{G}{2 r^{3}}+\frac{F}{2 r}-L\right) \Theta-\frac{\bar{\theta}}{2 \mu}\right\} \text { if } j=l-1 / 2
\end{aligned}
$$

here $\left(k_{+}(l), k_{-}(l)\right) \equiv \frac{1}{2}(l,-l-1), \quad j$ and $l$ are the total quantum number and orbital angular momentum quantum number, respectively. The non-null diagonal elements $\left(H_{\text {so }}^{\text {ciq }}\right)_{11}$ and $\left(H_{s o}^{c i q}\right)_{22}$ will affect the energy values $E_{n}$ by creating two new values $E_{\mathrm{u}}^{c i q}$ and $E_{\mathrm{d}}^{c i q}$, respectively. Details of these are given in the next subsection. After a profound calculation, 
it can be shown that the two new radial Schrödinger equations for $U_{n l}(r)$ and $\chi_{n l}(r)$ satisfy the following differential equations for MCIQP, respectively:

$$
\begin{array}{r}
\frac{d^{2} U_{n l}(r)}{d r^{2}}+\frac{2}{r} \frac{d U_{n l}(r)}{d r}+2 \mu\left(E_{n c}^{c i q}-V_{n c-e f f}^{c i q}\right) U_{n l}(r)=0 \\
\Rightarrow \frac{d^{2} \chi_{n l}(r)}{d r^{2}}+2 \mu\left(E_{n c}^{c i q}-V_{n c-e f f}^{c i q}\right) \chi_{n l}(r)=0
\end{array}
$$

The new effective potential for MCIQP $V_{n c-e f f}^{c i q}$ in (NC: 3D-RSP) symmetries is given by:

$$
V_{n c-e f f}^{c i q}=V_{e f f}^{c i q}-\left(\frac{C}{r^{4}}+\frac{G}{2 r^{3}}+\frac{F}{2 r}-L\right) \overrightarrow{\mathbf{L}} \vec{\Theta}+\frac{\overrightarrow{\mathbf{L}} \vec{\theta}}{2 \mu}
$$

Eq. (24) indicates that $H_{\text {per }}^{c i q}(r)$ is proportional to two infinitesimals parameters ( $\Theta$ and $\bar{\theta}$ ). Thus the modified radial part, that is, equation (28.1) is solved by applying the standard perturbation theory to find acceptable solutions at the first order of two parameters $\Theta$ and $\bar{\theta}$ - The proposed solutions for MSE under MCIQP include energy corrections. Those corrections are produced automatically from two principal physical phenomena, the first one is the effect of modified spin-orbit interaction and the second one is the modified Zeeman effect. Furthermore, the stark effect that appears in the linear part of MCIQP can also be observed.

\subsection{The exact modified spin-orbit spectrum for hydrogenic atoms under MCIQP model}

The purpose of this sub-section is to give a complete description of the determination of the energy level of hydrogenic atoms such as $\mathrm{He}^{+}, \mathrm{Be}^{+}$and $\mathrm{Li}^{2+}$ under MCIQP. To achieve this goal, we first find the corrections $E_{\mathrm{u}}^{c i q}$ and $E_{\mathrm{d}}^{c i q}$ for hydrogenic atoms. Those corrections have two polarities up and down corresponding to $j=l+1 / 2$ and $j=l-1 / 2$, respectively, at the first order of the two parameters $(\Theta$ and $\bar{\theta}$ ). This is obtained by applying the standard perturbation theory as follows:

$$
E_{\mathrm{u}}^{c i q}=\left\langle\Psi\left|\left(H_{s o}^{c i q}\right)_{11}\right| \Psi\right\rangle \text { and } E_{\mathrm{d}}^{c i q}=\left\langle\Psi\left|\left(H_{\text {so }}^{c \mathrm{iq}}\right)_{22}\right| \Psi\right\rangle
$$

By inserting $\int|\vec{r}\rangle\langle\vec{r}| d^{3} r=1$, the above equation is easily rewritten in the equivalent form:

$E_{u}^{c i q}=-\gamma C_{n l}^{2} k_{+} \int_{0}^{+\infty} r^{-\frac{N}{\sqrt{H_{n}}}+2} \exp \left(2 \sqrt{H_{n}} r\right)\left\{\left(-r^{2} \frac{d}{d r}\right)^{n}\left(r^{-2 n-\frac{N}{\sqrt{H_{n}}}} \exp \left(-2 \sqrt{H_{n}} r\right)\right)\right\}^{2}\left(\left(\begin{array}{l}\frac{C}{r^{4}}+\frac{G}{2 r^{3}} \\ +\frac{F}{2 r}-L\end{array}\right) \Theta-\frac{\bar{\theta}}{2 \mu}\right) d r$ 


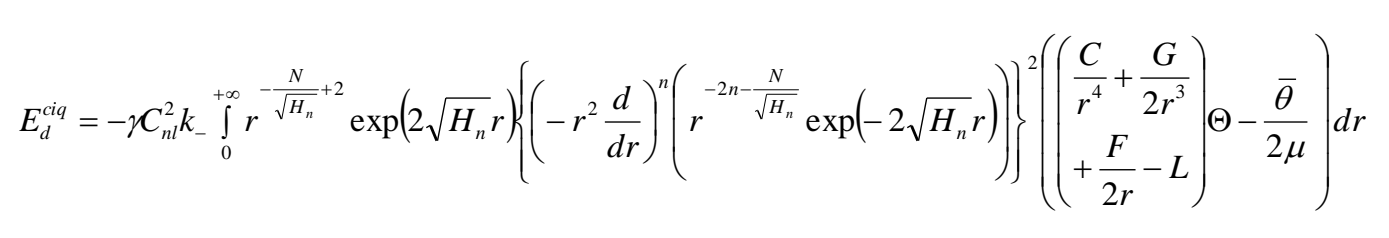

Here the orthogonality property of the spherical harmonics $\int Y_{l}^{m}(\theta, \varphi) Y_{l^{\prime}}^{m^{\prime}}(\theta, \varphi) \sin (\theta) d \theta d \varphi$ $=\delta_{l l} \delta_{m m^{\prime}}$ is used. Now, the above two equations can be further simplified to the new form: $E_{u}^{c i q}\left(k_{+}, C, G, F, L, n, N, H_{n}\right)=-\gamma C_{n l}{ }^{2} k_{+}\left\{\Theta\left[\begin{array}{l}T_{1}\left(C, n, N, H_{n}\right)+T_{2}\left(G, n, N, H_{n}\right) \\ +T_{3}\left(F, n, N, H_{n}\right)+T_{4}\left(L, n, N, H_{n}\right)\end{array}\right]-\frac{\bar{\theta}}{2 \mu} T_{5}\left(n, N, H_{n}\right)\right\}$
$E_{d}^{c i q}\left(k_{-}, C, G, F, L, n, N, H_{n}\right)=-\gamma C_{n l}{ }^{2} k_{-}\left\{\Theta\left[\begin{array}{l}T_{1}\left(C, n, N, H_{n}\right)+T_{2}\left(G, n, N, H_{n}\right) \\ +T_{3}\left(F, n, N, H_{n}\right)+T_{4}\left(L, n, N, H_{n}\right)\end{array}\right]-\frac{\bar{\theta}}{2 \mu} T_{5}\left(n, N, H_{n}\right)\right\}$

Moreover, the expressions of the five factors $T_{i}(i=\overline{1,5})$ are given by:

$$
\begin{aligned}
& \frac{T_{1}\left(C, n, N, H_{n}\right)}{C}=\int_{0}^{+\infty} r^{-\frac{N}{\sqrt{H_{n}}}-2} \exp \left(2 \sqrt{H_{n}} r\right)\left\{\left(-r^{2} \frac{d}{d r}\right)^{n}\left(r^{-2 n-\frac{N}{\sqrt{H_{n}}}} \exp \left(-2 \sqrt{H_{n}} r\right)\right)\right\}^{2} d r \\
& \frac{2 T_{2}\left(G, n, N, H_{n}\right)}{G}=\int_{0}^{+\infty} r^{-\frac{N}{\sqrt{H_{n}}}-1} \exp \left(2 \sqrt{H_{n}} r\right)\left\{\left(-r^{2} \frac{d}{d r}\right)^{n}\left(r^{-2 n-\frac{N}{\sqrt{H_{n}}}} \exp \left(-2 \sqrt{H_{n}} r\right)\right)\right\}^{2} d r \\
& \frac{2 T_{3}\left(F, n, N, H_{n}\right)}{F}=\int_{0}^{+\infty} r^{-\frac{N}{\sqrt{H_{n}}}+1} \exp \left(2 \sqrt{H_{n}} r\right)\left\{\left(-r^{2} \frac{d}{d r}\right)^{n}\left(r^{-2 n-\frac{N}{\sqrt{H_{n}}}} \exp \left(-2 \sqrt{H_{n}} r\right)\right)\right\}^{2} d r \\
& \frac{T_{4}\left(L, n, N, H_{n}\right)}{L}=-\int_{0}^{+\infty} r^{-\frac{N}{\sqrt{H_{n}}}+2} \exp \left(2 \sqrt{H_{n}} r\right)\left\{\left(-r^{2} \frac{d}{d r}\right)^{n}\left(r^{-2 n-\frac{N}{\sqrt{H_{n}}}} \exp \left(-2 \sqrt{H_{n}} r\right)\right)\right\}^{2} d r \\
& T_{5}\left(n, N, H_{n}\right)=\int_{0}^{+\infty} r^{-\frac{N}{\sqrt{H_{n}}}+2} \exp \left(2 \sqrt{H_{n}} r\right)\left\{\left(-r^{2} \frac{d}{d r}\right)^{n}\left(r^{-2 n-\frac{N}{\sqrt{H_{n}}}} \exp \left(-2 \sqrt{H_{n}} r\right)\right)\right\}^{2} d r
\end{aligned}
$$

For the ground state, the expressions of the five factors $T_{i}(i=\overline{1,5})$ can be simplified as follows:

$$
\begin{aligned}
& T_{1}\left(C, 0, N, H_{0}\right)=C \int_{0}^{+\infty} r^{-\frac{3 N}{\sqrt{H_{0}}}-1-1} \exp \left(-2 \sqrt{H_{0}} r\right) d r, T_{2}\left(G, 0, N, H_{0}\right)=\frac{G}{2} \int_{0}^{+\infty} r^{-\frac{3 N}{\sqrt{H_{0}}}-1} \exp \left(-2 \sqrt{H_{0}} r\right) d r \\
& T_{3}\left(F, 0, N, H_{0}\right)=\frac{F}{2} \int_{0}^{+\infty} r^{-\frac{3 N}{\sqrt{H_{0}}}+2-1} \exp \left(-2 \sqrt{H_{n}} r\right) d r, T_{4}\left(L, 0, N, H_{0}\right)=-L \int_{0}^{+\infty} r r^{-\frac{3 N}{\sqrt{H_{0}}}+3-1} \exp \left(-2 \sqrt{H_{0}} r\right) d r \\
& T_{5}\left(0, N, H_{0}\right)=\int_{0}^{+\infty} r^{-\frac{3 N}{\sqrt{H_{0}}}+3-1} \exp \left(-2 \sqrt{H_{0}} r\right) d r
\end{aligned}
$$


where $H_{0}=-2 \mu\left(E_{0}-D-3 F r_{0}+6 L r_{0}^{2}\right)$. It is convenient to apply the following special integral ${ }^{48}$ :

$$
\int_{0}^{+\infty} x^{\nu-1} \exp \left(-\beta x^{p}\right) d x=\frac{\beta^{-\frac{v}{p}}}{p} \Gamma\left(\frac{v}{p}\right)
$$

with conditions $(\operatorname{Re} \beta\rangle 0, \operatorname{Re} v>0$ and $p>0)$ while $\Gamma(v / p)=\int_{0}^{+\infty} \exp (-t) t^{(v / p)-1}$ is the ordinary Gamma function. After straightforward calculations, the following explicit results can be obtained:

$$
\begin{gathered}
T_{1}\left(C, 0, N, H_{0}\right)=C\left(2 \sqrt{H_{0}}\right)^{\frac{3 N}{\sqrt{H_{0}}}+1} \Gamma\left(-\frac{3 N}{\sqrt{H_{0}}}-1\right) \\
T_{2}\left(G, 0, N, H_{0}\right)=\frac{G}{2}\left(2 \sqrt{H_{0}}\right)^{\frac{3 N}{\sqrt{H_{0}}}} \Gamma\left(-\frac{3 N}{\sqrt{H_{0}}}\right) \\
T_{3}\left(F, 0, N, H_{0}\right)=\frac{F}{2}\left(2 \sqrt{H_{0}}\right)^{\frac{3 N}{\sqrt{H_{0}}}-2} \Gamma\left(2-\frac{3 N}{\sqrt{H_{0}}}\right) \\
\text { and } T_{4}\left(L, 0, N, H_{0}\right)=-L\left(2 \sqrt{H_{0}}\right)^{\frac{3 N}{\sqrt{H_{0}}}-3} \Gamma\left(3-\frac{3 N}{\sqrt{H_{0}}}\right)=-\mathrm{L} T_{5}\left(0, N, H_{0}\right)
\end{gathered}
$$

The exact modifications $E^{c i q}\left(k_{+}, C, G, F, L, n=0, N, H_{0}\right)$ and $E_{d}^{c i q}\left(k_{-}, C, G, F, L, n=0, N, H_{0}\right)$ of the ground state can be obtained as follows:

$$
\begin{aligned}
& E_{u}^{c i q}\left(k_{+}, C, G, F, L, 0, N, H_{0}\right)=-\gamma C_{00}^{2} k_{+}(l=0)\left\{\Theta T_{01}\left(C, 0, C, G, F, L, N, H_{0}\right)-\frac{\bar{\theta}}{2 \mu} T_{5}\left(0, N, H_{0}\right)\right\} \\
& E_{d}^{c i q}\left(k_{-}, C, G, F, L, 0, N, H_{0}\right)=-\gamma C_{00}^{2} k_{-}(l=0)\left\{\Theta T_{01}\left(C, 0, C, G, F, L, N, H_{0}\right)-\frac{\bar{\theta}}{2 \mu} T_{5}\left(0, N, H_{0}\right)\right\}
\end{aligned}
$$

with $T_{01}\left(C, 0, C, G, F, L, N, H_{0}\right)=T_{1}\left(C, 0, N, H_{0}\right)+T_{2}\left(G, 0, N, H_{1}\right)+T_{3}\left(F, 0, N, H_{0}\right)+T_{4}\left(L, 0, N, H_{0}\right)$. For the first excited state, the expressions of the five factors $T_{i}(i=\overline{1,5})$ are given by: 


$$
\begin{gathered}
T_{1}\left(C, n=1, N, H_{1}\right)=C \int_{0}^{+\infty}\left\{\begin{array}{l}
\alpha^{2} r^{2 \alpha+\lambda+1-1} \exp \left(-\beta_{1} r\right)+\beta_{1}{ }^{2} r^{2 \alpha+\lambda+3-1} \exp \left(-\beta_{1} r\right) \\
-2 \alpha \beta_{1} r^{2 \alpha+\lambda+2-1} \exp \left(-\beta_{1} r\right)
\end{array}\right\} d r \\
T_{2}\left(G, n=1, N, H_{1}\right)=\frac{G}{2} \int_{0}^{+\infty}\left\{\begin{array}{l}
\alpha^{2} r^{2 \alpha+\lambda+2-1} \exp \left(-\beta_{1} r\right)+\beta_{1}{ }^{2} r^{2 \alpha+\lambda+4-1} \exp \left(-\beta_{1} r\right) \\
-2 \alpha \beta_{1} r^{2 \alpha+\lambda+3-1} \exp \left(-\beta_{1} r\right)
\end{array}\right\} d r \\
T_{3}\left(F, n=1, N, H_{1}\right)=\frac{F}{2} \int_{0}^{+\infty}\left\{\begin{array}{l}
\alpha^{2} r^{2 \alpha+\lambda+4-1} \exp \left(-\beta_{1} r\right)+\beta_{1}{ }^{2} r^{2 \alpha+\lambda+6-1} \exp \left(-\beta_{1} r\right) \\
-2 \alpha \beta_{1} r^{2 \alpha+\lambda+5-1} \exp \left(-\beta_{1} r\right)
\end{array}\right\} d r \\
T_{4}\left(L, n=1, N, H_{1}\right)=-L \int_{0}^{+\infty}\left\{\begin{array}{l}
\alpha^{2} r^{2 \alpha+\lambda+5-1} \exp \left(-\beta_{1} r\right)+\beta_{1}{ }^{2} r^{2 \alpha+\lambda+7-1} \exp \left(-\beta_{1} r\right) \\
-2 \alpha \beta_{1} r^{2 \alpha+\lambda+6-1} \exp \left(-\beta_{1} r\right)
\end{array}\right\} d r \\
T_{5}\left(n=1, N=1, H_{1}\right)=\int_{0}^{+\infty}\left\{\begin{array}{l}
\alpha^{2} r^{2 \alpha+\lambda+5-1} \exp \left(-\beta_{1} r\right)+\beta_{1}{ }^{2} r^{2 \alpha+\lambda+7-1} \exp \left(-\beta_{1} r\right) \\
-2 \alpha \beta_{1} r^{2 \alpha+\lambda+6-1} \exp \left(-\beta_{1} r\right)
\end{array}\right\} d r
\end{gathered}
$$

where $H_{1}=-2 \mu\left(E_{1}-D-3 F r_{0}+6 L r_{0}^{2}\right), \quad \beta_{1}=2 \sqrt{H_{1}}, \quad \alpha=\lambda-2 \quad$ and $\quad \lambda=-\frac{N}{\sqrt{H_{1}}}$.

Evaluating, the integrals shown in Eqs. (36.1) and (36.2) by applying the special integral given by Eq. (33), the following results can be obtained as exact modifications $E_{\text {u }}^{c i q}\left(k_{+}, C, G, F, L, 1, N, H_{1}\right)$ and $E_{\mathrm{d}}^{c i q}\left(k_{-}, C, G, F, L, 1, N, H_{1}\right)$ :

$$
\begin{gathered}
T_{1}\left(C, 1, N, H_{1}\right)=C \beta_{1}^{-2 \alpha-\lambda-1}\left\{\alpha^{2} \Gamma(2 \alpha+\lambda+1)-2 \alpha \Gamma(2 \alpha+\lambda+2)+\Gamma(2 \alpha+\lambda+3)\right\} \\
T_{2}\left(G, 1, N, H_{1}\right)=\frac{G}{2} \beta_{1}^{-2 \alpha-\lambda-2}\left\{\alpha^{2} \Gamma(2 \alpha+\lambda+2)-2 \alpha \Gamma(2 \alpha+\lambda+3)+\Gamma(2 \alpha+\lambda+4)\right\} \\
T_{3}\left(F, 1, N, H_{1}\right)=\frac{F}{2} \beta_{1}^{-2 \alpha-\lambda-4}\left\{\alpha^{2} \Gamma(2 \alpha+\lambda+4)-2 \alpha \Gamma(2 \alpha+\lambda+5)+\Gamma(2 \alpha+\lambda+6)\right\} \\
T_{4}\left(L, 1, N, H_{1}\right)=-L \beta_{1}^{-2 \alpha-\lambda-5}\left\{\alpha^{2} \Gamma(2 \alpha+\lambda+5)-2 \alpha \Gamma(2 \alpha+\lambda+6)+\Gamma(2 \alpha+\lambda+7)\right\} \\
=-L T_{5}\left(1, N=1, H_{1}\right)
\end{gathered}
$$

As mentioned previously, this energy is produced with the effect of induced spin-orbit interaction at the first excited state as follows:

$$
\begin{aligned}
& E_{u}^{c i q}\left(k_{+}, C, G, F, L, 1, N, H_{1}\right)=-\gamma C_{n l}^{2} k_{+}\left\{\Theta T_{11}\left(1, C, G, F, L, N, H_{1}\right)-\frac{\bar{\theta}}{2 \mu} T_{5}\left(1, N, H_{1}\right)\right\} \\
& E_{d}^{c i q}\left(k_{-}, C, G, F, L, 1, N, H_{1}\right)=-\gamma C_{n l}^{2} k_{-}\left\{\Theta T_{11}\left(1, C, G, F, L, N, H_{1}\right)-\frac{\bar{\theta}}{2 \mu} T_{5}\left(1, N, H_{1}\right)\right\}
\end{aligned}
$$

Where the factor $T_{11}\left(1, C, G, F, L, N, H_{1}\right)=T_{1}\left(C, 1, N, H_{1}\right)+T_{2}\left(G, 1, N, H_{1}\right)+T_{3}\left(F, 1, N, H_{1}\right)+T_{4}\left(L, 1, N, H_{1}\right)$. Furthermore, in the same manner as before, the exact modifications $E_{u}^{c i q}\left(k_{+}, C, G, F, L, n, N, H_{n}\right)$ and $E_{d}^{c i q}\left(k_{+}, C, G, F, L, n, N, H_{n}\right)$ for $n^{\text {th }}$ excited states can be found as follows: 


$$
\begin{aligned}
& E_{u}^{c i q}\left(k_{+}, C, G, F, L, n, N, H_{1}\right)=-\gamma C_{n l}^{2} k_{+}\left\{\Theta T_{1 n}\left(n, C, G, F, L, N, H_{n}\right)-\frac{\bar{\theta}}{2 \mu} T_{5}\left(n, N, H_{n}\right)\right\} \\
& E_{d}^{c i q}\left(k_{-}, C, G, F, L, n, N, H_{1}\right)=-\gamma C_{n l}^{2} k_{-}\left\{\Theta T_{1 n}\left(n, C, G, F, L, N, H_{n}\right)-\frac{\bar{\theta}}{2 \mu} T_{5}\left(n, N, H_{n}\right)\right\}
\end{aligned}
$$

where $T_{1 n}=T_{1}\left(C, n, N, H_{n}\right)+T_{2}\left(G, n, N, H_{n}\right)+T_{3}\left(F, n, N, H_{n}\right)+T_{4}\left(L, n, N, H_{n}\right)$ and $T_{5}\left(n, N, H_{n}\right)=-L T_{4}\left(L, n, N, H_{n}\right)$. The result shown in the Eq. (39) is specific to hydrogenic atoms such as $\mathrm{He}^{+}, \mathrm{Be}^{+}$and $\mathrm{Li}^{2+}$.

\subsection{The modified magnetic spectrum for hydrogenic atoms under MCIQP model}

In addition to the important results obtained previously, another important physically meaningful phenomenon produced by the effect of MCIQP related to the influence of an external uniform magnetic field $\vec{B}$ is considered. Moreover, to achieve this without repeating the previous calculations, it is sufficient to apply the following replacements:

$$
\vec{\Theta} \rightarrow \chi \vec{B} \quad \text { and } \quad \overrightarrow{\bar{\theta}} \rightarrow \vec{\sigma} \vec{B}
$$

to make the following changes.

$$
\left\{-\left(\frac{C}{r^{4}}+\frac{G}{2 r^{3}}+\frac{F}{2 r}-L\right) \vec{\Theta} \vec{L}+\frac{\vec{\theta} \vec{L}}{2 \mu}\right\} \rightarrow\left\{-\left(\frac{C}{r^{4}}+\frac{G}{2 r^{3}}+\frac{F}{2 r}-L\right) \chi+\frac{\bar{\sigma}}{2 \mu}\right\} \vec{B} \vec{L}
$$

Here $\chi$ and $\bar{\sigma}$ are two infinitesimal real proportional constants. The arbitrary uniform external magnetic field $\vec{B}$ is chosen to be parallel to the $(\mathrm{Oz})$ axis. The new modified magnetic Hamiltonian $H_{m}^{c i q}(r, \chi, \bar{\sigma})$ in (NC: 3D-RSP) symmetries can be written as:

$$
H_{\mathrm{so}}^{c \mathrm{iq}}(r, \Theta, \bar{\theta}) \rightarrow H_{m q}^{\mathrm{ciq}}(r, \chi, \bar{\sigma})=-\left\{\left(\frac{C}{r^{4}}+\frac{G}{2 r^{3}}+\frac{F}{2 r}-L\right) \chi-\frac{\bar{\sigma}}{2 \mu}\right\}\left(\vec{B} \vec{J}-\aleph_{z}\right)
$$

here $\aleph_{z} \equiv-\vec{S} \vec{B}$ denotes the Zeeman effect in commutative quantum mechanics, while $\aleph_{\bmod -z} \equiv \vec{B} \vec{J}-\aleph_{z}$ is the new Zeeman effect. Now, to obtain the exact NC magnetic modifications of energy $E_{m}^{c i q}\left(m=0, C, G, F, L, n=0, N, H_{0}\right), E_{m}^{c i q}\left(m=0, \pm 1, C, G, F, L, n=1, N, H_{1}\right)$ and $E_{m}^{c i q}\left(m=\overline{-l,+l}, C, G, F, L, n, N, H_{n}\right) \quad$ corresponding to the ground state, the first excited state and the $n^{\text {th }}$ excited states, respectively, of hydrogenic atoms such as $\mathrm{He}^{+}, \mathrm{Be}^{+}$and $\mathrm{Li}^{2+}$, replace one of two factors $\left(k_{+}\right.$or $k_{-}$) in the Eqs. (35) and (38) by the magnetic quantum number $m$ and the infinitesimal parameter $\Theta$ by the new infinitesimal coefficient $\chi$. Thus, the following results can be obtained: 


$$
\begin{aligned}
& E_{m}^{c i q}\left(m=0, C, G, F, L, 0, N, H_{0}\right)=0 \\
& E_{m}^{c i q}\left(m=0, \pm 1, C, G, F, L, 1, N, H_{1}\right)=-\gamma C_{1 l}^{2}\left\{\chi T_{11}\left(1, C, G, F, L, N, H_{1}\right)-\frac{\bar{\sigma}}{2 \mu} T_{5}\left(1, N, H_{1}\right)\right\} B m \\
& E_{m}^{c i q}\left(m=\overline{-l,+l}, C, G, F, L, n, N, H_{n}\right)=-\gamma C_{1 l}^{2}\left\{\chi T_{1 n}\left(1, C, G, F, L, N, H_{1}\right)-\frac{\bar{\sigma}}{2 \mu} T_{5}\left(1, N, H_{1}\right)\right\} B m
\end{aligned}
$$

Since $-l \leq m \leq+l,(2 l+1)$ values can be fixed for the discreet number $m$. It should be noted that the results obtained in Eq. (42) can be found by direct calculation $E_{m}^{c i q}=\left\langle\Psi \mid H_{m}^{c i q}(r, \chi, \bar{\sigma}) \Psi\right\rangle$ that makes the following explicit relation:

$E_{m}^{c i q}=-\gamma C_{n l}^{2} m B \int_{0}^{+\infty} r^{-\frac{N}{\sqrt{H_{n}}}+2} \exp \left(2 \sqrt{H_{n}} r\right)\left\{\left(-r^{2} \frac{d}{d r}\right)^{n}\left(r^{-2 n-\frac{N}{\sqrt{H_{n}}}} \exp \left(-2 \sqrt{H_{n}} r\right)\right)\right\}^{2}\left(\left(\begin{array}{l}\frac{C}{r^{4}}+\frac{G}{2 r^{3}} \\ +\frac{F}{2 r}-L\end{array}\right) \chi-\frac{\bar{\sigma}}{2 \mu}\right) d r$

This can be rewritten as the equivalent form:

$$
E_{m}^{c i q}=-\gamma C_{n l}^{2} m B\left\{\chi\left[\begin{array}{l}
T_{1}\left(C, n, N, H_{n}\right)+T_{2}\left(G, n, N, H_{n}\right) \\
+T_{3}\left(F, n, N, H_{n}\right)+T_{4}\left(L, n, N, H_{n}\right)
\end{array}\right]-\frac{\bar{\sigma}}{2 \mu} T_{5}\left(n, N, H_{n}\right)\right\}
$$

Then the corrections produced by the Hamiltonian operator $H_{m}^{c i q}(r, \chi, \bar{\sigma})$ for the ground state and other excited states can be found by repeating the same calculations in the previous subsection.

\section{GLOBAL SPECTRUM OF THE HYDROGENIC ATOMS UNDER MCIQP AT FINITE TEMPERATURE:}

In the previous sections, the solution of the MSE for the hydrogenic atoms such as ( $\mathrm{He}^{+}, \mathrm{Be}^{+}$and $\mathrm{Li}^{2+}$ ) under the MCIQP model was obtained as Eq. (22) by using the generalized Bopp's shift method and standard perturbation theory. The corrections $E_{(\mathrm{u}-\mathrm{d})}^{c i q}\left(k_{ \pm}, C, G, F, L, 0, N, H_{0}\right), \quad E_{(\mathrm{a}-\mathrm{d})}^{c i q}\left(k_{ \pm}, C, G, F, L, 1, N, H_{0}\right) \quad$ and $E_{(\mathrm{u}-\mathrm{d})}^{c i q}\left(k_{ \pm}, C, G, F, L, n, N, H_{0}\right)$ have also been obtained. Two polarities up and down corresponding $j=l+1 / 2$ and $j=l-1 / 2$, for the ground state, the first excited state and the generalized excited states, respectively have also been seen. Those corrections were produced with the effect of induced spin-orbit operator and the modified Zeeman effect operator in the (NC: 3D-RSP) symmetries. Now, the modified eigenenergies $\left(E_{n c}^{u c i q}, E_{n c}^{d c i q}\right)\left(0, m=0, C, G, F, L, N, H_{0}\right)$, $\left(E_{n c}^{u c i q}, E_{n c}^{d c i q}\right)\left(1, m=0, \pm 1, C, G, F, L, N, H_{1}\right)$ and $\left(E_{n c}^{u c i q}, E_{n c}^{d c i q}\right)\left(n, m=\overline{-l,+l}, C, G, F, L, N, H_{n}\right)$ with spin-1/2 based on our original results, which were presented as Eqs. (35), (38), (39) and (42) can easily be deduced, in addition to the energy $E_{n l}$ for CIQP in the Eq. (12.1), as follows: 


$$
\begin{aligned}
& E_{n c}^{u c i q}\left(0, m=0, C, G, F, L, N, H_{0}\right)=E_{0} \\
& E_{n c}^{c d i q}\left(0, m=0, C, G, F, L, N, H_{0}\right)=E_{0}-\gamma C_{0 l}^{2} k_{+}(l=0)\left\{\begin{array}{l}
\Theta T_{01}\left(C, 0, C, G, F, L, N, H_{0}\right) \\
-\frac{\bar{\theta}}{2 \mu} T_{5}\left(0, N, H_{0}\right)
\end{array}\right\} \\
& E_{n c}^{u c i q}\left(1, k_{+},(m=0, \pm 1), C, G, F, L, N, H_{1}\right)=E_{1}-\gamma C_{1 l}^{2}\left(\begin{array}{l}
\left(k_{+} \Theta+\chi B m\right) T_{11}\left(1, C, G, F, L, N, H_{1}\right) \\
-\left(\frac{\bar{\theta}}{2 \mu} k_{+}+\frac{\bar{\sigma}}{2 \mu} B m\right) T_{5}\left(1, N, H_{1}\right)
\end{array}\right\} \\
& E_{n c}^{d c i q}\left(1, k_{-},(m=0, \pm 1), C, G, F, L, N, H_{1}\right)=E_{1}-\gamma C_{1 l}^{2}\left(\begin{array}{l}
\left(k_{-} \Theta+\chi B m\right) T_{11}\left(1, C, G, F, L, N, H_{1}\right) \\
-\left(\frac{\bar{\theta}}{2 \mu} k_{-}+\frac{\bar{\sigma}}{2 \mu} B m\right) T_{5}\left(1, N, H_{1}\right)
\end{array}\right\} \\
& E_{n c}^{u c i q}\left(n, k_{+}, m=\overline{-l,+l}, C, G, F, L, N, H_{n}\right)=E_{n l}-\gamma C_{n l}^{2}\left\{\begin{array}{l}
\left(k_{+} \Theta+\chi B m\right) T_{1 n}\left(n, C, G, F, L, N, H_{n}\right) \\
-\left(\frac{\bar{\theta}}{2 \mu} k_{+}+\frac{\bar{\sigma}}{2 \mu} B m\right) T_{5}\left(n, N, H_{n}\right)
\end{array}\right\}(4) \\
& E_{n c}^{d c i q}\left(n, k_{-}, m=\overline{-l,+l}, C, G, F, L, N, H_{n}\right)=E_{n l}-\gamma C_{n l}^{2}\left\{\begin{array}{l}
\left(k_{-} \Theta+\chi B m\right) T_{1 n}\left(n, C, G, F, L, N, H_{n}\right) \\
-\left(\frac{\bar{\theta}}{2 \mu} k_{+}+\frac{\bar{\sigma}}{2 \mu} B m\right) T_{5}\left(n, N, H_{n}\right)
\end{array}\right\}
\end{aligned}
$$

Where the two values of energies $E_{0}$ and $E_{1}$ of the ground state and the first excited state are given as follows:

$$
\begin{aligned}
& E_{0}=D+\frac{3 F}{\delta}-\frac{6 L}{\delta^{2}}-\frac{1}{2 \mu}\left[\frac{2 \mu\left(\frac{3 F}{\delta^{2}}-\frac{8 L}{\delta^{3}}+G\right)}{1 \pm \sqrt{1+\frac{8 \mu F}{\delta^{3}}-24 \frac{L}{\delta^{4}}+8 \mu C}}\right]^{2} \\
& E_{1}=D+\frac{3 F}{\delta}-\frac{6 L}{\delta^{2}}-\frac{1}{2 \mu}\left[\frac{2 \mu\left(\frac{3 F}{\delta^{2}}-\frac{8 L}{\delta^{3}}+G\right)}{3 \pm \sqrt{1+4 l(l+1)+\frac{8 \mu F}{\delta^{3}}-24 \frac{L}{\delta^{4}}+8 \mu C}}\right]^{2}
\end{aligned}
$$

This is one of the main objectives of our research. Note that the obtained eigenvalues of energies are real and the NC diagonal Hamiltonian $H_{n c}^{c i q}\left(x_{\mu}, p_{\mu}\right)$ is Hermitian. Furthermore, it is possible to write the three elements $\left(H_{n c}^{c i q}\right)_{11},\left(H_{n c}^{c i q}\right)_{22}$ and $\left(H_{n c}^{c i q}\right)_{33}$ as follows:

$$
H_{c i q}\left(x_{\mu}, p_{\mu}\right) \rightarrow H_{n c}^{c i q}\left(x_{\mu}, p_{\mu}\right) \equiv \operatorname{diag}\left(\left(H_{n c}^{c i q}\right)_{11},\left(H_{n c}^{c i q}\right)_{22},\left(H_{n c}^{c i q}\right)_{33}\right)
$$

Where $\left(H_{n c}^{c i q}\right)_{11}=-\frac{\Delta_{n c}}{2 \mu}+H_{\text {int }}^{u c i q},\left(H_{n c}^{c i q}\right)_{22}=-\frac{\Delta_{n c}}{2 \mu}+H_{\text {int }}^{d c i q}$ and $\left(H_{n c}^{c i q}\right)_{33}=-\frac{\Delta}{2 \mu}+V_{c i q}(r)$ while the new kinetic term is given by:

$$
\frac{\Delta}{2 \mu} \rightarrow \frac{\Delta_{n c}}{2 \mu}=\frac{\Delta-\vec{\theta} \vec{L}-\vec{\sigma} \vec{L}}{2 \mu}
$$


and the two modified interactions elements $\left(H_{\mathrm{int}}^{u c i q}, H_{\mathrm{int}}^{\text {dciq }}\right)$ are given by:

$$
\left\{\begin{array}{l}
H_{\text {int }}^{u c i q}=A(T, r) r-\frac{B(T, r)}{r}+C(T, r) r^{2}-\gamma k_{+}(l)\left\{\left(\frac{C}{r^{4}}+\frac{G}{2 r^{3}}+\frac{F}{2 r}-L\right) \Theta-\frac{\bar{\theta}}{2 \mu}\right\} \\
-\left\{\left(\frac{C}{r^{4}}+\frac{G}{2 r^{3}}+\frac{F}{2 r}-L\right) \chi-\frac{\bar{\sigma}}{2 \mu}\right\} \aleph_{\text {mod }-z} \\
H_{\text {int }}^{d c i q}=(T, r) r-\frac{B(T, r)}{r}+C(T, r) r^{2}-\gamma k_{-}(l)\left\{\left(\frac{C}{r^{4}}+\frac{G}{2 r^{3}}+\frac{F}{2 r}-L\right) \Theta-\frac{\bar{\theta}}{2 \mu}\right\} \\
-\left\{\left(\frac{C}{r^{4}}+\frac{G}{2 r^{3}}+\frac{F}{2 r}-L\right) \chi-\frac{\bar{\sigma}}{2 \mu}\right\} \aleph_{\text {mod }-z}
\end{array}\right.
$$

Thus, the kinetic term for CIQP model $\left(-\frac{\Delta}{2 \mu}\right)$ and the interaction term appear in the Eq. (6), are replaced by a new modified form of kinetic term $\frac{\Delta_{n c}}{2 \mu}$ and new modified interactions ( $H_{\mathrm{int}}^{u c i q}$ and $H_{\mathrm{int}}^{d c i q}$ ) in (NC-3D: RSP) symmetries. On the other hand, it is evident to consider the quantum number $m$ takes $(2 l+1)$ values and the global momentum operator takes two values $l+\frac{1}{2}$ and $l+\frac{1}{2}$. Thus, every state in usual three-dimensional space of energy for hydrogenic atoms under MCIQP will become double $2(2 l+1)$ sub-states. To obtain the total complete degeneracy of energy levels of hydrogenic atoms in (NC-3D: RSP) symmetries, all allowed values of $l$ are to be added. Total degeneracy is thus,

$$
\underbrace{2 \sum_{l=0}^{n-1}(2 l+1)}_{\text {NRQM }} \equiv 2 n^{2} \rightarrow \underbrace{2\left(\sum_{l=0}^{n-1} 2(2 l+1)\right)}_{\text {NRNCQM }} \equiv 4 n^{2}
$$

Thus, the total complete degeneracy of the energy level of the MCIQP model will be doubled. This gives a very good indicator that our new treatment produces clear energy values when compared with similar energy levels obtained in NRQM. Note that the obtained energy eigenvalues $\left(E_{n c}^{u c i q}, E_{n c}^{d c i q}\right)\left(n,(m=\overline{-l,+l}), C, G, F, L, N, H_{n}\right)$ now depend on new discrete atomic quantum numbers $(n, j, l, s)$ and $m$ in addition to the parameters ( $C, G, F, L, N)$ of the potential. 


\section{GLOBAL SPECTRUM OF THE QUARKONIUM SYSTEMS UNDER MCIQP MODEL AT FINITE TEMPERATURE:}

The second main principle goal of this work is to treat the case of systems with spin$1 / 2$, for example, the quarkonium systems, such as the charmonium $c \bar{c}$, bottomonium $b \bar{b}$ and mesons $c \bar{s}$. It is well known that the eigenvalues $j$ of total operator $\vec{J}$ can be obtained from the interval $|l-s| \leq j \leq|l+s|$. This allows us to obtain the eigenvalues of the coupling operator $\vec{L} \vec{S}$ as $k(j, l, s) \equiv j(j+1)-l(l+1)-s(s+1)$. Then the nonrelativistic energy spectrum $E_{n c}^{c i q}\left(n, k(j, l, s),(m=\overline{-l,+l}), C, G, F, L, N, H_{n}\right)$ of the quarkonium systems can be obtained directly from the Eq. (44.3). We need to replace one of three factors ( $k_{+}$or $k_{-}$and $\gamma)$ by the new two factor $k(j, l, s)$ and $g_{s}$ which represent the strong coupling in the QCD theory. This is to avoid repeating previous calculations. This allows us to get the following results:

$$
\begin{aligned}
E_{n c}^{c i q}\left(n, k(j, l, s),(m=\overline{-l,+l}), C, G, F, L, N, H_{n}\right)=E_{n l}- \\
-g_{s} C_{n l}^{2}\left\{\begin{array}{l}
(k(, l, s) \Theta+\chi B m) T_{1 n}\left(n, C, G, F, L, N, H_{n}\right) \\
-\left(\frac{\bar{\theta}}{2 \mu} k(j, l, s)+\frac{\bar{\sigma}}{2 \mu} B m\right) T_{5}\left(n, N, H_{n}\right)
\end{array}\right\}
\end{aligned}
$$

Our last application is to calculate the mass spectra of the heavy quarkonium system such as charmonium, bottomonium that have the quark and antiquark flavor and mesons $c \bar{s}$ under MCIQP model at finite temperature. To achieve this goal first the mass formula of quarkonium in 3-dimensional space is recalled ${ }^{49-51}$ :

$$
M=2 m_{q}+E_{n l}
$$

In order to achieve this goal, the traditional formula $M=2 m_{q}+E_{n l}$ is generalized to the new form:

$$
M=2 m_{q}+E_{n l} \rightarrow M_{n c}^{c i q}=2 m_{q}+E_{n c}^{c i q}\left(n, k(j, l, s), m, C, G, F, L, N, H_{n}\right)
$$

here $m_{q}$ is a bare mass of quarkonium or twice the reduced mass of the system and $E_{n c}^{c i q}$ is the energy in NRNCQM symmetries. Thus, at finite temperature $T \neq 0$ for the modified mass of quarkonium systems $\quad M_{n c}^{c i q}$, the following equation is obtained:

$$
M_{n c}^{c i q}=2 m_{q}+g_{s} C_{n l}^{2}\left\{\begin{array}{l}
\left(\frac{\bar{\theta}}{2 \mu} k(j, l, s)+\frac{\bar{\sigma}}{2 \mu} B m\right) T_{5}\left(n, N, H_{n}\right) \\
-(k(j, l, s) \Theta+\chi B m) T_{1 n}\left(n, C, G, F, L, N, H_{n}\right)
\end{array}\right\}
$$


here $M$ is the heavy quarkonium system at a finite temperature under CIQP in NRQM ${ }^{1}$. Now, the Eq. (49) is applied on the charmonium, bottomonium and mesons $c \bar{s}$. It is well known that the spin of charmonium, bottomonium and mesons $c \bar{s}$ are equal to two values ( 0 or 1 ). For the case of spin-1, from the interval $|l-1| \leq j \leq|l+1|$, three values of $j(l-1, l$ and $l+1)$ are provided. These three values are allowed to be fixed as $\left(k_{1}(l), k_{2}(l), k_{3}(l)\right) \equiv \frac{1}{2}(l,-2,-2 l-2)$. Thus, the three values of energy are given by.

$$
\begin{gathered}
E_{n c}^{c i q}\left(k_{1}(l), n, l, m, C, G, F, L, N, H_{n}\right)=E_{n l}-g_{s} C_{n l}^{2}\left(\begin{array}{l}
\left(k_{1}(l) \Theta+\chi B m\right) T_{1 n}\left(n, l, C, G, F, L, N, H_{n}\right) \\
-\left(\frac{\bar{\theta}}{2 \mu} k_{1}(l)+\frac{\bar{\sigma}}{2 \mu} B m\right) T_{5}\left(n, N, H_{n}\right)
\end{array}\right\} \\
E_{n c}^{c i q}\left(k_{2}(l), n, l, m, C, G, F, L, N, H_{n}\right)=E_{n l}-g_{s} C_{n l}^{2}\left\{\begin{array}{l}
\left(k_{2}(l) \Theta+\chi B m\right) T_{1 n}\left(n, l, C, G, F, L, N, H_{n}\right) \\
-\left(\frac{\bar{\theta}}{2 \mu} k_{2}(l)+\frac{\bar{\sigma}}{2 \mu} B m\right) T_{5}\left(n, N, H_{n}\right)
\end{array}\right\} \\
E_{n c}^{c i q}\left(k_{3}(l), n, l, m, C, G, F, L, N, H_{n}\right)=E_{n l}-g_{s} C_{n l}^{2}\left\{\begin{array}{l}
\left(k_{3}(l) \Theta+\chi B m\right) T_{1 n}\left(n, l, C, G, F, L, N, H_{n}\right) \\
-\left(\frac{\bar{\theta}}{2 \mu} k_{3}(l)+\frac{\bar{\sigma}}{2 \mu} B m\right) T_{5}\left(n, N, H_{n}\right)
\end{array}\right\}
\end{gathered}
$$

Thus, the modified mass of the charmonium $c \bar{c}$, bottomonium $b \bar{b}$ and mesons $c \bar{s}$ becomes:

$$
M=2 m_{q}+E_{n l} \rightarrow M_{n c}^{c i q}=M+\frac{1}{3}\left(\begin{array}{l}
E_{n c}^{c i q}\left(k_{1}=l / 2\right)+E_{n c}^{c i q}\left(k_{2}=-1\right) \\
+E_{n c}^{c i q}\left(k_{3}=-l-1\right)
\end{array}\right)\left(n, m, C, G, F, L, N, H_{n}\right)
$$

here $\frac{1}{3}\left(E_{n c}^{c i q}\left(k_{1}=l / 2\right)+E_{n c}^{c i q}\left(k_{2}=-1\right)+E_{n c}^{c i q}\left(k_{3}=-l-1\right)\right)$ are the non-polarized energies (energy independent of spin). For the case of spin- $0, j$ is equal only to one value of $l$, which allows the null values of $k(j, l, s)$ to be obtained. Thus, the modified mass of quarkonium system $M_{n c}^{c i q}$ can be taken as the following new result:

$$
M_{n c}^{c i q}\left(n, l, m, C, G, F, L, N, H_{n}\right)=M\left(C, G, F, L, N, H_{n}\right)+g_{s} C_{n l}^{2}\left\{\begin{array}{lll}
M_{1} & \text { for } & \text { spin - } 1 \\
M_{2} & \text { for } & \text { spin-0 }
\end{array}\right.
$$

Here, the two perturbative masses $M_{1}(\vec{S}=\overrightarrow{1})$ and $M_{2}(\vec{S}=\overrightarrow{0})$ are given by: 


$$
\begin{aligned}
& M_{1}(\vec{S}=\overrightarrow{1})=\left(\chi B m+\frac{l+4}{6} \Theta+\right) T_{1 n}\left(n, C, G, F, L, N, H_{n}\right)-\left(\frac{\bar{\sigma}}{2 \mu} B m+(l+4) \frac{\bar{\theta}}{12 \mu}\right) T_{5}\left(n, N, H_{n}\right) \\
& M_{2}(\vec{S}=\overrightarrow{0})=\left\{\chi T_{1 n}\left(n, C, G, F, L, N, H_{n}\right)+\frac{\bar{\sigma}}{2} T_{5}\left(n, N, H_{n}\right)\right\} B m
\end{aligned}
$$

Finally, the contribution for the hydrogenic atoms, such as $\left(\mathrm{He}^{+}, \mathrm{Be}^{+}\right.$and $\left.\mathrm{Li}^{2+}\right)$ is obtained. The ordinary energy $E_{n l}$ in NRNCQM will be modified by two values $\delta E_{u p}$ and $\delta E_{d o w n}$, which correspond to the two polarities up and down, in the symmetries of NRNCQM, as follows:

$$
\left\{\begin{array}{l}
\delta E_{u p} \equiv-\gamma C_{n l}^{2}\left\{\left(k_{+} \Theta+\chi B m\right) T_{1 n}\left(n, C, G, F, L, N, H_{n}\right)-\left(\frac{\bar{\theta}}{2 \mu} k_{+}+\frac{\bar{\sigma}}{2 \mu} B m\right) T_{5}\left(n, N, H_{n}\right)\right\} \\
\text { for - polarity: up } \\
\delta E_{\text {down }} \equiv-\gamma C_{n l}^{2}\left\{\left(k_{-} \Theta+\chi B m\right) T_{1 n}\left(n, C, G, F, L, N, H_{n}\right)-\left(\frac{\bar{\theta}}{2 \mu} k_{+}+\frac{\bar{\sigma}}{2 \mu} B m\right) T_{5}\left(n, N, H_{n}\right)\right\} \\
\text { for - polarity: down }
\end{array}\right.
$$

Moreover, for the quarkonium system such as the charmonium $c \bar{c}$, bottomonium $b \bar{b}$ and mesons $c \bar{s}$, the ordinary energy $E_{n l}$ will be modified by new additive part $\delta E_{n c}^{c i q}$, in the symmetries of NCQM. This modification is given by:

$$
\delta E_{n c}^{c i q} \equiv-g_{s} C_{n l}^{2}\left\{\begin{array}{l}
(k(, l, s) \Theta+\chi B m) T_{1 n}\left(n, C, G, F, L, N, H_{n}\right) \\
-\left(\frac{\bar{\theta}}{2 \mu} k(j, l, s)+\frac{\bar{\sigma}}{2 \mu} B m\right) T_{5}\left(n, N, H_{n}\right)
\end{array}\right\}
$$

here $\delta E_{n c}^{c i q}$ represents the difference between the two values $E_{n c}^{c i q}\left(n, k(j, l, s), m=-\bar{l},+l, C, G, F, L, N, H_{n}\right)$ and $E_{n l}$.

\section{MAIN RESULTS}

The present work is divided into two-fold. The goal of the first part was to find a solution of the MSE for the hydrogenic atoms such as $\left(\mathrm{He}^{+}, \mathrm{Be}^{+}\right.$and $\left.\mathrm{Li}^{2+}\right)$ under MCIQP using the generalized Bopp's shift method and standard perturbation theory. The energy eigenvalue is calculated in the (NC: 3D-RSP) symmetries. The modified eigenenergies $\left(E_{n c}^{u c i q}, E_{n c}^{d c i q}\right)\left(0, m=0, C, G, F, L, N, H_{0}\right), \quad\left(E_{n c}^{u c i q}, E_{n c}^{d c i q}\right)\left(1, m \equiv 0, \pm 1, C, G, F, L, N, H_{1}\right) \quad$ and $\left.\left(E_{n c}^{u c i q}, E_{n c}^{d c i q}\right)\left(n, m \equiv \overline{-l,+l}, C, G, F, L, N, H_{n}\right)\right)$ and corresponding Hamiltonian operator $H_{n c}^{c i q}\left(x_{\mu}, p_{\mu}\right)$ are obtained. In the second part, the eigenvalues expressions for the quarkonium systems such as the charmonium $c \bar{c}$, bottomonium $b \bar{b}$ and mesons $c \bar{s}$ are 
obtained. It should be noted that our results in recent work are in good agreement with the results already exist in literature in NCQM where the physical treatment was done using other potentials such as new modified potential containing Cornell, Gaussian and inverse square terms ${ }^{40}$ and modified quark-antiquark interaction potential ${ }^{52}$. If $(\Theta, \bar{\theta}) \rightarrow(0,0)$ is considered, the results of the commutative space of first reference ${ }^{40}$ obtained for the Cornell plus inverse quadratic potential at finite temperature can be reproduced. This limiting condition reproduces the results exist in literature.

\section{CONCLUSION}

This paper is devoted to solving the MSE for the modified Cornell plus inverse quadratic potential at finite temperature. Our work has been organized around two major parts. In the first part, nonrelativistic spectrum of hydrogenic atoms such as $\mathrm{He}^{+}, \mathrm{Be}^{+}$and $\mathrm{Li}^{2+}$ which interacted with this potential at finite temperature was considered. The energy spectra of the quarkonium systems, such as the charmonium $c \bar{c}$, bottomonium $b \bar{b}$ and mesons $c \bar{s}$ were considered in the second part. The generalized Bopp's shift method and standard perturbation theory in the (NC: 3D-RSP) symmetries were applied; the main results obtained are summarized below.

- Ordinary interaction $\left(A(T, r) r-\frac{B(T, r)}{r}+C(T, r) r^{2}\right)$ in NRQM was replaced by new modified interactions according to the results shown in Eq. (47),

- The ordinary kinetic term was modified to the new form in Eq. (46.1) for heavy quarkonium systems and hydrogenic atoms under the influence of MCIQP model,

- The perturbative corrections for the ground state, the first excited state and the generalized excited states with spin-1/2 and spin $\neq 1 / 2$ have been obtained as results shown in Eqs. (44.1), (44.2), (44.3), respectively. In addition, the energy for heavy quarkonium systems under the influence of MCIQP was shown in Eq. (49),

- The modified mass of quarkonium systems $M_{n c}^{c i q}$ for spin- ( 0 or 1$)$ have been obtained at finite temperature $(T \neq 0)$. The mass values were equal to the sum of the corresponding value $M$ in NRQM and two perturbative terms proportional to two parameters $(\Theta$ or $\chi)$ and $(\bar{\theta}$ or $\bar{\sigma})$,

- The MCIQP was suggested as an effective potential for quark-antiquark interaction at finite temperature. It describes hydrogenic atoms because the global potential contains a Coulomb potential term in the (NC: 3D-RSP) symmetries.

- The energy eigenvalues corresponding to $(\Theta, \bar{\theta}) \rightarrow(0,0)$ which were obtained here can be reduced to the result obtained in Ref. ${ }^{1}$ for the SE in three-dimension subjected to the Cornell plus inverse quadratic potential at finite temperature.

The important results of this article are the ability and capability of the MSE to play a vital role in calculating and describing many phenomena as in high-energy physics (HEP). The 
solutions of the MSE are important for calculating the mass of quarkonia such as charmonium $c \bar{c}$, bottomonium $b \bar{b}$, and mesons $c \bar{s}$ with spin (0 or 1) under MCIQP at finite temperature in (NC: 3D-RSP) symmetries.

Here a new theoretical model in the field of (NC: 3D-RSP) symmetries has been presented. Furthermore, energy values which appeared quantitative and interesting have been obtained. This can be considered as a revolution at the theoretical level. Presently, technological applications for this model are being worked out.

\section{ACKNOWLEDEMENTS}

Anonymous referees are acknowledged for their useful comments and suggestions which greatly improved the paper. The Algerian Ministry of Higher Education and Scientific Research and DGRST are also acknowledged for the partial support given under Project No. B00L02UN280120180001. The help extended by Professors H. Baaziz and Z. Charifi to improve this paper is much appreciated.

\section{REFERENCES}

1. A.I. Ahmadov, C. Aydin and O. Uzun, Bound stat solution of the Schrödinger equation at finite temperature. IOP Conf. Series: Journal of Physics: Conf. Series. 1194, (2019) 012001. DOI: https://doi:10.1088/1742-6596/1194/1/012001.

2. M. Abu-Shady, T.A. Abdel-Karim and E.M. Khokha, Binding Energies and Dissociation Temperatures of Heavy Quarkonia at Finite Temperature and Chemical Potential in the $\mathrm{N}$ Dimensional Space. Advances in High Energy Physics Volume 2018, Article ID 7356843, 12 pages.. DOI: https://doi.org/10.1155/2018/7356843.

3. M. Abu-Shady, N-dimensional Schrödinger equation at finite temperature using the Nikiforov-Uvarov method. Journal of the Egyptian Mathematical Society. 25(1), (2017) 8689.. DOI: https:// doi:10.1016/j.joems.2016.06.006.

4. S.M. Kuchin and N.V. Maksimenko, Theoretical Estimations of the Spin Averaged Mass Spectra of Heavy Quarkonia and Bc Mesons. Univ. J. Phys. Appl. 1, (2013) 295. DOI: https://DOI:10.13189/ujpa.2013.010310.

5. A. Al-Jamel and H. Widyan, Heavy quarkonium mass spectra in a coulomb field plus quadratic potential using Nikiforov-Uvarov method. Appl. Phys. Res. 4, (2012) 94. DOI: https://DOI:10.5539/apr.v4n3p94.

6. S. Capozziello, G. Lambiase and G. Scarpetta, Generalized uncertainty principle from quantum geometry. Int. J. Theor. Phys. 39, (2000) 15. DOI: https://doi.org/10.1023/A:1003634814685.

7. E. Passos, L.R. Ribeiro, C. Furtado, Noncommutative Anandan quantum phase. Phys Rev A 76, (2007) 012113. DOI: https://doi.org/10.1103/PhysRevA.76.012113. 
8. L.R. Ribeiro, E. Passos, C. Furtado and J.R. Nascimento, Geometric phases modified by a Lorentz-symmetry violation background. International Journal of Modern Physics A. 30(14) (2015) 1550072. DOI: https://doi: 10.1142/s0217751x15500724.

9. Ö.F. Dayi, Dynamics of dipoles and quantum phases in noncommutative coordinates. EPL (Europhysics Letters). 85(4), (2009) 41002. DOI: https://doi:10.1209/0295-5075/85/41002.

10. Ö.F. Day and B. Yapışkan, An alternative formulation of Hall effect and quantum phases in noncommutative space. Physics Letters A. 374(37), (2010) 3810-3817. DOI: https://doi:10.1016/j.physleta.2010.07.043.

11. W. Heisenberg, in Letter of Heisenberg to Peierls (1930), Wolfgang Pauli, Scientific Correspondence, edited by von K. Meyenn, Springer-Verlag, Berlin, 1993.

12. H. Snyder, Quantized Space-Time. Phys. Rev. 71(1), (1947) 38-41. DOI: https://doi:10.1103/physrev.71.38.

13. P.M. Ho and H.C. Kao, Noncommutative Quantum Mechanics from Noncommutative Quantum Field Theory. Physical Review Letters 88(15), (2002). DOI: https://doi:10.1103/physrevlett.88.151602.

14. M. Darroodi, H. Mehraban and H. Hassanabadi, The Klein-Gordon equation with the Kratzer potential in the noncommutative space. Modern Physics Letters A. 33 (35), (2018) 1850203. DOI: https://doi : 10.1142/s0217732318502036.

15. Abdelmadjid Maireche, Solutions of Two-dimensional Schrodinger Equation in Symmetries of Extended Quantum Mechanics for the Modified Pseudoharmonic Potential: an Application to Some Diatomic Molecules. J. Nano- Electron. Phys. 11(4), (2019) 04013. DOI: https://doi.org/10.21272/jnep.11 (4).04013.

16. P. Gnatenko. Parameters of noncommutativity in Lie-algebraic noncommutative space. Physical Review D. 99(2), (2019) 026009-1. DOI: https://doi:10.1103/physrevd.99.026009.

17. Abdelmadjid Maireche, The Klein-Gordon Equation with Modified Coulomb Potential plus Inverse-Square-Root Potential in Three-Dimensional Noncommutative Space. To Physics Journal.3 (2019) 186-196. Retrieved from

https://purkh.com/index.php/tophy/article/view/489.

18. P. Gnatenko and V.M. Tkachuk, Weak equivalence principle in noncommutative phase space and the parameters of noncommutativity. Physics Letters A. 381(31), (2017) 24632469. DOI: https://doi:10.1016/j.physleta.2017.05.056.

19. O. Bertolami, J.G. Rosa, C.M.L. De aragao, P. Castorina and D. Zappala, Scaling of varialbles and the relation between noncommutative parameters in noncommutative quantum mechanics. Modern Physics Letters A. 21(10), (2006) 795-802. DOI: https://doi: $10.1142 / \mathrm{s} 0217732306019840$.

20. Abdelmadjid Maireche, A Recent Study of Excited Energy Levels of Diatomics for Modified more General Exponential Screened Coulomb Potential: Extended Quantum Mechanics. J. Nano- Electron. Phys. 9(3), (2017) 03021. DOI: https://doi: 10.21272/jnep.9 (3).03021.

21. E.F. Djemaï and H. Smail, On Quantum Mechanics on Noncommutative Quantum Phase Space. Commun. Theor. Phys. (Beijing, China). 41(6), (2004) 837-844. DOI: https://doi:10.1088/0253-6102/41/6/837. 
22. Yi YUAN, LI Kang, WANG Jian-Hua and CHEN Chi-Yi. Spin-1/2 relativistic particle in a magnetic field in NC phase space. Chinese Physics C. 34(5), (2010) 543-547.. DOI: https://doi:10.1088/1674-1137/34/5/005.

23. O. Bertolami and P. Leal. Aspects of phase-space noncommutative quantum mechanics. Physics Letters B. 750, (2015) 6-11. DOI: https:// doi:10.1016/j.physletb.2015.08.024.

24. C. Bastos, O. Bertolami, N.C. Dias and J.N. Prata, Weyl-Wigner formulation of noncommutative quantum mechanics. Journal of Mathematical Physics. 49(7), (2008) 072101. DOI: https://doi:10.1063/1.2944996.

25. J. Zhang, Fractional angular momentum in non-commutative spaces. Physics Letters B. 584(1-2), (2004) 204-209. DOI: https:// doi:10.1016/j.physletb.2004.01.049.

26. J. Gamboa, M. Loewe and J.C. Rojas, Noncommutative quantum mechanics. Phys. Rev. D. 64, (2001) 067901. DOI: https://doi.org/10.1103/PhysRevD.64.067901.

27. M. Chaichian, Sheikh-Jabbari and A. Tureanu, Hydrogen Atom Spectrum and the Lamb Shift in Noncommutative QED. Physical Review Letters. 86(13), (2001) 2716-2719. DOI: https://doi:10.1103/physrevlett.86.2716.

28. Abdelmadjid Maireche, New Relativistic Atomic Mass Spectra of Quark ( $u, d$ and s) for Extended Modified Cornell Potential in Nano and Plank's Scales. J. Nano- Electron. Phys. 8(1), (2016) 01020-1 - 01020-7. DOI: https://doi 10.21272/jnep.8(1).01020.

29. J. Wang and K. Li, The HMW effect in noncommutative quantum mechanics. Journal of Physics A: Mathematical and Theoretical. 40(9), (2007) 2197-2202. DOI: https://doi:10.1088/1751-8113/40/9/021.

30. Abdelmadjid Maireche, New Bound State Energies for Spherical Quantum Dots in Presence of a Confining Potential Model at Nano and Plank's Scales. NanoWorld J. 1(4), (2016) 122129. DOI: https://doi: 10.17756/nwj.2016-016.

31. K. Li and J. Wang, The topological AC effect on non-commutative phase space. The European Physical Journal C. 50(4), (2007) 1007-1011. DOI: https://doi:10.1140/epic/s10052-007-0256-0.

32. L.I. Schiff, Quantum Mechanics, (McGraw- Hill, New York, 1968), 3rd Edition.

33. L.D. Landau and E. M. Lifshitz, Quantum Mechanics: Non-Relativistic Theory, (Pergamon, New York 1977), 3rd Edition.

34. Abdelmadjid Maireche. A Complete Analytical Solution of the Mie-Type Potentials in Noncommutative 3-Dimensional Spaces and Phases Symmetries. Afr. Rev Phys. 11, (2016) 111117.

35. Abdelmadjid Maireche, A New Nonrelativistic Investigation for the Lowest Excitations States of Interactions in One-Electron Atoms, Muonic, Hadronic and Rydberg Atoms with Modified Inverse Power Potential. International Frontier Science Letters. 9, (2016) 33-46. DOI : https://doi.org/10.18052/www.scipress.com/IFSL.9.33.

36. Abdelmadjid Maireche, New quantum atomic spectrum of Schrödinger equation with pseudo harmonic potential in both noncommutative three-dimensional spaces and phases. Lat. Am. J. Phys. Educ. 9(1), (2015)1301. 
37. Abdelmadjid Maireche, New Bound States for Modified Vibrational-Rotational Structure of Supersingular plus Coulomb Potential of the Schrödinger Equation in One-Electron Atoms. International Letters of Chemistry, Physics and Astronomy.73, (2017) 31-45. DOI: https://doi.org/10.18052/www.scipress.com/ILCPA.73.31

38. Abdelmadjid Maireche. Extended of the Schrödinger Equation with New Coulomb Potentials plus Linear and Harmonic Radial Terms in the Symmetries of Noncommutative Quantum Mechanics. J. Nano- Electron. Phys. 10(6) (2018) 06015-1 - 06015-7. DOI: https://doi.org/10.21272/jnep.10(6).06015.

39. Abdelmadjid Maireche, Investigations on the Relativistic Interactions in One-Electron Atoms with Modified Yukawa Potential for Spin 1/2 Particles. International Frontier Science Letters. 11, (2017) 29. DOI : https://doi.org/10.18052/www.scipress.com/IFSL.11.29.

40. Abdelmadjid Maireche, Nonrelativistic treatment of Hydrogen-like and neutral atoms subjected to the generalized perturbed Yukawa potential with centrifugal barrier in the symmetries of noncommutative Quantum mechanics. International Journal of Geometric Methods in Modern Physics.17(05), (2020) 2050067. DOI: https:// DOI: $10.1142 / \mathrm{S} 021988782050067 \mathrm{X}$.

41. Abdelmadjid Maireche, The Klein-Gordon equation with modified Coulomb plus inversesquare potential in the noncommutative three-dimensional space. Modern Physics Letters A. 35(5), (2020) 2050015. DOI: https://Doi: 10.1142/s0217732320500157.

42. Abdelmadjid Maireche, New Nonrelativistic Three-Dimensional Spectroscopic Studies of NMGECSC Potential in Presence of External Electric. J. Nano- Electron. Phys. 10(4), (2018). 04003 DOI: http://dx.doi.org/10.21272/jnep.10(4).04003.

43. Abdelmadjid Maireche, Effects of Three-Dimensional Noncommutative Theories on Bound States Schrödinger Molecular under New Modified Kratzer-type Interactions. J. NanoElectron. Phys. 10(2), (2018) 02011. DOI: https://doi.org/10.21272/jnep.10(2).02011.

44. M.A. De Andrade and C. Neves, Noncommutative mapping from the symplectic formalism. Journal of Mathematical Physics. 59(1), (2018) 012105. DOI: https://doi:10.1063/1.4986964.

45. E.M.C. Abreu, C. Neves, and W. Oliveira, Noncommutativity from the symplectic point of view. Int. J. Mod. Phys. A 21, (2006) 5359. DOI: https://doi: 10.1142/s0217751x06034094.

46. E.M.C. Abreu, J.A. Neto, A.C.R. Mendes, C. Neves, W. Oliveira and M.V. Marcial, Lagrangian formulation for noncommutative nonlinear systems. Int. J. Mod. Phys. A. 27, (2012) 1250053. DOI: https://doi: 10.1142/s0217751x12500534.

47. L. Mezincescu, Star operation in quantum mechanics. High Energy Physics-Theory (hepth), (2000), viewed $21 \mathrm{Jul}$ 2000, arXiv:hep-th/0007046.

48. I.S. Gradshteyn and I.M. Ryzhik, Table of Integrals, Series and Products, edited by Alan Jeffrey (University of Newcastle upon Tyne, England and Daniel Zwillinger, Rensselaer Polytechnic Institute USA 2007), 7th. Ed p. 370. 
49. M. Abu-Shady, T.A. Abdel-Karim and S.Y. Ezz-Alarab, Masses and thermodynamic properties of heavy mesons in the non-relativistic quark model using the Nikiforov-Uvarov method. J Egypt Math Soc. 27: 14, (2019) 1-15. DOI: https://doi.org/10.1186/s42787-0190014-0.

50. M. Abu-Shady and S.Y. Ezz-Alarab. Trigonometric Rosen-Morse Potential as a QuarkAntiquark Interaction Potential for Meson Properties in the Non-relativistic Quark Model Using EAIM. Few-Body Systems. 60(4), (2019). DOI: https://doi: 10.1007/s00601-0191531-y.

51. N.V. Maksimenko and S.M. Kuchin. Electric polarizability of pions in semirelativistic quark model. Physics of Particles and Nuclei Letters. 9(2), (2012) 134-138. DOI: https://Doi: $10.1134 / \mathrm{s} 1547477112020112$.

52. Abdelmadjid Maireche, A New Asymptotic Study to the 3-Dimensional Radial Schrödinger Equation under Modified Quark-antiquark Interaction Potential. J Nanosci Curr Res 4(1), (2019) 131. 